\title{
EFECTO DEL ENCUADRE SOBRE EL DESCUENTO TEMPORAL EN JÓVENES QUE CONSUMEN ALCOHOL: ANÁLISIS DEL DOMINIO
}

FRAMING EFFECT ON DELAY DISCOUNTING IN YOUNG ADULTS REPORTING ALCOHOL CONSUMPTION: ANALYSIS OF REWARD DOMAIN

Jorge L. Valenzuela-Reyes

Universidad Autónoma de Aguascalientes

Hugo E. Reyes-Huerta CONACYT - Universidad Autónoma de Aguascalientes

Cristiano V. dos Santos

Universidad de Guadalajara

Marina L. González-Torres Universidad Autónoma de Aguascalientes

Cómo citar este texto:

Valenzuela-Reyes, J.L., Reyes-Huerta, H.E., dos Santos, C.V. y González-Torres, M.L. (2022). Efecto del encuadre sobre el descuento temporal en jóvenes que consumen alcohol: análisis del dominio. Health and Addictions / Salud y Drogas, 22(1), 79-94. doi: 10.21134/haaj.v22i1.616 


\title{
Resumen
}

Favorecer la preferencia por beneficios futuros se ha logrado al hacer explícito que los resultados de una elección implican no recibir la alternativa no elegida, hallazgo denominado efecto del cero escondido. Objetivo. El presente estudio evaluó la generalidad del efecto en tareas de descuento por demora en dos dominios (i.e., monetario y salud) con participantes que reportaron consumo de alcohol. Método. Los participantes fueron expuestos a dos tipos de tareas, una con el cero implícito y otra con el cero explícito, esto en ambos dominios y empleando dos recompensas de distinta magnitud. Resultados. Los resultados mostraron una tendencia en la que el grado de descuento incrementa en función del consumo de alcohol, al menos para el caso de consecuencias monetarias. Además, se replicó el efecto del cero escondido y el efecto de magnitud independientemente del nivel de consumo de alcohol en tareas monetarias. No obstante, ninguno de estos efectos se observó en el dominio de salud con el procedimiento empleado. Adicionalmente, no se observó una asociación sistemática de la tasa de descuento con las consecuencias negativas vinculadas al consumo de alcohol. Conclusiones. Hacer explícito el costo de oportunidad resulta en una disminución de la tasa de descuento de recompensas monetarias, pero se requiere mayor investigación para precisar la ausencia del efecto en situaciones que involucran decisiones sobre la salud.

\begin{abstract}
Preference for delayed rewards has been promoted by making explicit that preference for one alternative implies not receiving the not chosen alternative, a result named the hidden-zero effect. Objective. The present research assessed the generality of the hidden-zero effect in delay discounting tasks in two domains (i.e., monetary and health) in participants that reported alcohol consumption. Method. The participants were exposed to two discounting tasks, one with the implicit zero and one with the explicit zero, using both health and monetary rewards and employing different amounts. Results. Results showed a tendency to reduce the value of future monetary consequences at higher rate as a function of alcohol drinking. Also, the hidden-zero effect and the magnitude effect were replicated in the monetary domain independently of alcohol drinking status. Nevertheless, none of these effects were observed using health outcomes with the actual procedure. Finally, no systematic association between discounting rate and negative consequences derived from alcohol consumption was observed. Conclusions. Making explicit the opportunity cost reduce discounting rate of monetary outcomes, but more research is needed to clarify the absence of effects when deciding about health.
\end{abstract}

\section{Palabras clave}

Descuento por Demora, Efecto de Encuadre, Efecto del Cero, Dominio, Salud.

\section{Keywords}

Delay Discounting, Framing Effect, Hidden-Zero Effect, Domain, Health. 


\section{Efecto del encuadre sobre el descuento temporal en jóvenes que consumen alcohol: análisis del dominio}

El consumo de alcohol es un problema de carácter mundial, pues cerca de 2, 348 millones de personas mayores de 15 años son bebedores de alcohol. Consumir alcohol es una conducta problemática que influye en el desarrollo de consecuencias negativas para la persona, tales como inestabilidad económica y emocional, violencia, problemas de salud, accidentes e incluso la muerte (World Health Organization, 2018). El problema del consumo y sus consecuencias pueden entenderse como conductas que se derivan de una serie de elecciones impulsivas (Gerst et al., 2017; Mitchell, 2019). Desde esta perspectiva, la evidencia sugiere que las personas que consumen alcohol tienden a mostrar mayores niveles de impulsividad que personas sin historial de consumo (Adams et al., 2017). Específicamente, los problemas relacionados con el consumo de alcohol y otras drogas se han asociado a la marcada preferencia por recompensas inmediatas derivada del poco valor atribuido al futuro (Amlung et al., 2017; Snider et al., 2019), propiedad de la conducta impulsiva conocida como descuento por demora o descuento temporal.

El descuento por demora muestra la disminución del valor de una recompensa en función del tiempo de espera a su obtención (Odum et al., 2020). Este mecanismo explica cómo el comportamiento puede ser poco sensible a los beneficios de eventos futuros favoreciendo la preferencia por beneficios inmediatos (Scholten et al., 2019). Así, las personas que consumen alcohol suelen ser poco sensibles a los beneficios de la abstinencia o al consumo controlado. Evidencia de lo anterior son los resultados que muestran que las personas con consumo de alcohol (1) descuentan más que personas sin historial de consumo (Field et al., 2007; Myerson et al., 2005; Takahashi et al., 2009); (2) personas con consumo controlado tienen un menor descuento que personas con consumo excesivo (Adams et al., 2017; Bailey et al., 2018; Gerst et al., 2017; Mayhew et al., 2019; Stam et al., 2020); (3) una mayor tasa de descuento se asocia a no buscar tratamiento en personas con historial de consumo de alcohol y comorbilidades (Gowin et al., 2018); (4) estrategias para promover el autocontrol reducen el grado de descuento y son efectivas para disminuir el consumo de alcohol (Athamneh et al., 2021); (5) se ha reportado una menor probabilidad de éxito terapéutico conforme el paciente descuenta más recompensas futuras (Moraleda et al., 2018 ); (6) en el mismo sentido, el menor grado de descuento suele asociarse a mayor número de días en abstinencia (Athamneh, 2019), entre otros aspectos. Por estos hallazgos, es importante conocer los factores que reducen la tasa de descuento y que, por tanto, pueden incidir en la conducta adictiva.

Una de las manipulaciones que ha mostrado reducir la tasa de descuento es hacer explícito el costo de oportunidad. El descuento temporal ha sido estudiado a partir de una elección inter-temporal en apariencia sencilla. En este tipo de elecciones, los participantes indican su preferencia por recibir una recompensa pequeña-inmediata o una recompensa grande-demorada. De esta manera, la elección de una de las alternativas supone no recibir la otra, aunque esto no necesariamente es explícito. No obstante, cuando se hace explícito (agregando un cero) que elegir la recompensa inmediata implica no recibir la recompensa demorada (e.g., \$1000 ahora y \$0 en 1 mes) y elegir la recompensa demorada implica no recibir la recompensa inmediata (e.g., elegir ganar \$2000 en 1 mes y \$0 ahora), se ha observado una reducción en la tasa de descuento, resultado denominado efecto del cero escondido (Magen et al., 2008) ${ }^{1}$. Hacer explícito el cero, que constituye un caso de efecto de encuadre ${ }^{2}$, ha sido replicado con distintos procedimientos (Koffarnus \& Bickel, 2014), con tareas que miden el descuento temporal de eventos pasado (Koffarnus et al., 2014; Radu et al., 2011) y cuando el cero se agrega a una sola de las alternativas (Wu \& He, 2012). No obstante, este efecto no ha sido observado en todos los estudios en que se ha evaluado, cuestionándose su generalidad (Naudé et al., 2018).

Evaluar la generalidad del encuadre del cero (implícito/explícito) en distintas poblaciones y situaciones puede ayudar a determinar la relevancia clínica de la estrategia (Scholten et al., 2019). La generalidad del efecto puede probarse con (1) personas que consumen alcohol y (2) en contextos no monetarios (i.e., salud). El primer aspecto permitiría

1 Para mayor precisión, se hará una distinción entre cero explícito (la manipulación propuesta) y cero implícito (situación tradicional).

2 El denominado efecto de encuadre (framing en inglés) muestra que las decisiones de las personas son afectadas por la manera en que las situaciones de elección se describen, aunque los parámetros (e.g., magnitud de la recompensa) no cambien. 
evaluar si personas con distinto nivel de consumo de alcohol son sensibles al costo de oportunidad cuando es explícito; es posible que las diferencias observadas en función del consumo se reduzcan al tener en consideración las implicaciones de una elección inter-temporal. La segunda manipulación permitiría evaluar la generalidad del encuadre del cero en dominios no monetarios, dado que se han observado que consecuencias de salud se descuentan menos (Odum et al., 2020), es posible que el resultado obedezca a que existe una mejor ponderación de las implicaciones de elegir una alternativa, de ser este el caso, es posible que el efecto cero no ocurra en contextos de salud.

Asimismo, puede ser importante evaluar el efecto cero con recompensas de distinta magnitud. Aun es poco claro por qué hacer explicita la no recepción de la alternativa no elegida reduce la tasa de descuento. Si el efecto en cuestión es causado por una ponderación distinta derivada de considerar el costo de oportunidad de no recibir la recompensa demorada (mayor magnitud), emplear recompensas más grandes debería influir la manipulación del cero explícito.

Adicionalmente, la mayor cantidad de estudios sobre impulsividad han relacionado el descuento con la cantidad/ frecuencia de consumo. Un propósito más en la presente investigación es explorar si el nivel de impulsividad se relaciona con la aparición de consecuencias negativas. Una posibilidad es que tanto el consumo de alcohol como las consecuencias negativas deriven de la conducta impulsiva. Otra posibilidad, es que la interacción entre la cantidad de consumo y el nivel de impulsividad produzca las consecuencias negativas, particularmente cuando existe un problema de consumo establecido. El presente estudio pretende explorar la primera posibilidad, es decir, si jóvenes que presentan consumo y tienen mayores niveles de impulsividad son más propensos a involucrarse en situaciones que deriven en consecuencias negativas (e.g., problemas legales) aun cuando no haya un problema de consumo establecido.

Así, explicitar el cero en las alternativas ha mostrado reducir la tasa de descuento. En personas que consumen alcohol, explicitar la no recepción de la recompensa inmediata podría incrementar la preferencia por dicha consecuencia propiciando una mayor tasa de descuento, o simplemente ignorar el costo de las decisiones a pesar de ser explícito, propiciando que la tasa de descuento no cambie. Por el contrario, si el efecto cero se replica en esta población, podría identificarse una forma de incrementar la conducta autocontrolada. Adicionalmente, no se conoce si el efecto se presentará en un dominio de salud, tal evaluación permitiría conocer no solo la generalidad del efecto, sino también identificar el proceso de toma de decisiones de personas con distintos niveles de consumo de alcohol en situaciones que involucran directamente consecuencias que afectan la salud.

\section{Método}

\section{Participantes}

Participaron 66 jóvenes con una media de 20.35 años (DE = 2), 35 mujeres y 31 hombres. Los participantes fueron estudiantes activos de distintas carreras de una universidad pública en México, quienes accedieron a participar en el estudio voluntariamente.

\section{Materiales e Instrumentos}

Para medir la tasa de descuento, se emplearon computadoras de escritorio en las que se presentó la tarea de descuento programada con el software Unityß. Adicionalmente, de manera impresa se entregó una versión acotada del cuestionario individual de la Encuesta Nacional de Drogas, Alcohol y Tabaco (2016) para recabar información socio-demográfica y sobre el consumo de sustancias. Así, con el instrumento se obtuvo información general de los participantes (e.g., sexo, edad, escolaridad, estado civil, entre otros) y se evaluó el consumo de alcohol en el último año y en el último mes. Los participantes indicaron su respuesta con una " $X$ " señalando la frecuencia de consumo sobre algún tipo de bebida (e.g., tres o más veces al día, dos veces al día, tres o cuatro veces a la semana, etc.) y la cantidad con una frecuencia específica (e.g., 4 copas tres o cuatro veces a la semana). Asimismo, los participantes indicaban con una " $\mathrm{X}$ " opciones que describían la frecuencia/cantidad del consumo de tabaco y de drogas ilegales. 
También se aplicó el instrumento Young Adult Alcohol Problem Screening Test (YAAPST) adaptado para México (Salazar et al., 2012), el cual permitió medir posibles consecuencias negativas asociadas al consumo de alcohol. El instrumento obtuvo un alpha de Cronbach de 0.82 en el apartado para toda la vida y 0.85 en los últimos 6 meses, así como 0.87 para el puntaje de severidad (frecuencia de ocurrencia en los últimos 6 meses). El instrumento consta de 19 preguntas sobre consecuencias negativas del consumo de alcohol que sucedieron alguna vez en la vida y en los últimos 6 meses. Para las consecuencias que sucedieron alguna vez en la vida, las opciones de respuestas eran sí o no; en el apartado para las ocurridas en los últimos seis meses, se requería especificar la cantidad de ocasiones en que sucedieron ( 0 veces, 1 vez, 2 veces, 3 veces, de 4 a 6 veces, de 7 a 11 veces, de 12 a 20 veces, más de 21 veces). Las 19 preguntas estaban distribuidas en cuatro factores: (1) consecuencias físicas, sociales y económicas; (2) consecuencias asociadas al rol e interpersonales; (3) consecuencias asociadas a la adicción; (4) transgresión de las normas legales y convencionales.

\section{Procedimiento}

El contacto con los participantes se realizó a partir de una invitación en el salón de clases, momento en el que se explicó en términos generales el tipo de estudio, el tiempo que requeriría su participación y en el que se proporcionaron los datos para contactar al investigador. A los interesados se les agendó una cita en las instalaciones del Departamento de Psicología, donde contestaron los instrumentos de evaluación y las tareas programadas por computadora. En función de las respuestas sobre consumo de alcohol, los participantes fueron asignados a tres grupos: sin consumo de alcohol, con consumo controlado y consumo excesivo ${ }^{3}$. Así, el día de la sesión experimental, los participantes leyeron un consentimiento informado sobre el experimento en el que accedieron a participar. Posterior a la firma del consentimiento, se les entregó un bolígrafo y los instrumentos impresos, para finalmente responder las tareas de descuento por demora en una computadora.

\section{Tarea de descuento temporal}

Para medir el grado de descuento se utilizó una tarea denominada de ajuste de magnitud, inicialmente descrita por Du et al. (2002). Los participantes debían indicar su preferencia por una recompensa demorada de valor fijo o por una recompensa inmediata de menor magnitud, del 50\% en el primer ensayo. A partir de la elección del participante, en los ensayos posteriores la recompensa inmediata aumentaba o disminuía dependiendo de la elección hecha: elegir la recompensa demorada aumentaba el valor de la inmediata y elegir la recompensa inmediata disminuía el valor de esta. El ajuste del valor de la recompensa inmediata iba disminuyendo la mitad del cambio en el ensayo previo (i.e., 25\%, 12.5\% y así sucesivamente) durante 6 ensayos. El procedimiento de ajuste se realizó con cinco demoras diferentes ( 1 mes, 3 meses, 6 meses, 1 año y 4 años) para obtener un punto de indiferencia para cada una de ellas ${ }^{4}$. Con los cinco puntos de indiferencia puede medirse el grado en que una recompensa pierde su valor $y$ evidenciar el grado de descuento.

\section{Condiciones Experimentales}

La tarea de descuento fue adaptada para evaluar el encuadre del cero implícito/explícito en los dominios de dinero y salud. Así, un tipo de tarea describía las situaciones como tradicionalmente se ha hecho (cero implícito) y el segundo

3 El nivel de consumo de alcohol se identificó a partir de los criterios señalados en la Norma Oficial Mexicana (NOM-047SSA2-2015) y la Organización Mundial de la Salud. Según la OMS el consumo moderado es el consumo menor a 60 gramos de alcohol por ocasión de consumo. En consonancia, para la NOM el nivel de consumo de alcohol en hombres mayores de 18 años debe ser menor a 13 gramos de por hora, 52 gramos por ocasión y no más de 156 gramos por semana. Para el caso de las mujeres mayores de 18 años, el consumo debe ser menor de 13 gramos de alcohol por hora, menos de 39 gramos de alcohol por ocasión y no mayor a 117 gramos por semana.

4 Un punto de indiferencia es el valor que asume la recompensa inmediata y que es considerado equivalente al valor de una recompensa que se obtiene después de cierta demora. Los puntos de indiferencia reflejan el valor descontado de una recompensa por el tiempo a su obtención. 
tipo de tarea hacia explícito el cero de la alternativa no elegida (cero explícito). Ambas tareas fueron presentadas con dos recompensas económicas $(\$ 2,000 \text { y } \$ 15,000)^{5}$ y con dos consecuencias asociadas a la salud (12 meses de salud y 48 meses de salud) para evaluar el efecto de magnitud ${ }^{6}$. Cada participante fue asignado de manera aleatoria aun orden diferente de presentación de las tareas. Al final, cada participante respondió ocho tareas de descuento temporal en una sola sesión experimental ( 2 tipos de tarea $\times 2$ dominios $\times 2$ magnitudes).

\section{Ejecución de la tarea de descuento}

En la tarea con recompensas monetarias, a los participantes se les presentaban una primera pantalla con instrucciones. En términos generales, las instrucciones indicaban que se les mostraría una serie de preguntas hipotéticas que deberían responder como en la vida real. Se señalaba que la tarea consistía en elegir entre una recompensa de dinero grande-demorada o una recompensa pequeña-inmediata. Asimismo, se precisaba que cada respuesta era independiente de anteriores y subsecuentes enfatizándose que no había respuestas correctas o incorrectas. Desde la segunda pantalla los participantes empezaban a indicar su preferencia ante las situaciones, donde en la parte superior se les hacia la pregunta “¿Qué prefieres?” y en la parte media de la pantalla aparecían las opciones de respuesta, mismas que para ser seleccionadas debían ser "arrastradas" a unos recuadros vacíos. En la parte inferior los participantes debían confirmar su respuesta presionando el botón "continuar".

Así, para las tareas monetarias con cero implícito, a los participantes se les mostraba la siguiente situación: (A) Ganar $\$ 15,000$ en 1 mes $O(B)$ Ganar $\$ 7,500$ ahora. Para la tarea monetaria con cero explícito, la situación presentada era la siguiente: (A) Ganar \$15, 000 en 1 mes y \$0 ahora o (B) Ganar \$7, 500 ahora y \$0 en un mes. En ambas tareas, los participantes arrastraban la opción preferida a los recuadros vacíos y confirmaban su preferencia, en seguida se presentaba un nuevo ensayo con el ajuste de la magnitud correspondiente. Después de seis ensayos se identificaba un punto de indiferencia y la demora a la recompensa grande era sustituida y se proseguía hasta obtener una función de descuento.

En la tarea de salud, la primera pantalla solo difería en que la situación hipotética se trataba de tratamientos (Tx) que devolverán la salud por meses, tanto con cero implícito como explícito. Una segunda pantalla indicaba una situación hipotética de enfermedad que los participantes debían imaginar que tenían, la situación fue adaptada de Chapman (1996) (ver Apéndice). Finalmente, en una tercera pantalla los participantes elegían entre tratamientos que devolvían la salud por meses bajo la misma estructura que con recompensas monetarias (Tx [A] 48 meses de salud iniciando en 1 mes y 0 meses de salud del Tx [B] que iniciarían hoy o Tx [B] 24 meses de salud que inician hoy y 0 meses de salud del Tx [A] que iniciarían en un mes).

\section{Análisis de los datos}

Para evaluar si las respuestas de los participantes fueron sistemáticas en la tarea de descuento por demora, en el experimento se realizó un análisis de datos siguiendo los criterios de Johnson y Bickel (2008). Comúnmente, se cataloga un dato como asistemático si cumple con el criterio de que algún punto de indiferencia fuese mayor que el anterior por un $20 \%$ del valor de la recompensa estándar (criterio 1), o bien, si el último punto de indiferencia no fue menor en $10 \%$ que el primer punto de indiferencia (criterio 2 ).

Para medir el grado de descuento de las recompensas se calculó el área bajo la curva (AUC, por sus siglas en inglés) propuesta por Myerson et al., (2001) como una medida sin compromiso teórico. En este caso, la medida arroja valores entre 0 y 1 (valores normalizados), valores cercanos a 0 demuestran mayor grado de descuento.

Con relación a las consecuencias negativas, para obtener la media de cada uno de los cuatro factores antes mencio-

5 Pesos mexicanos.

6 El efecto de magnitud hace referencia al hecho de que recompensas grandes se descuentan menos que recompensas pequeñas. 
nados, los participantes contestaban a preguntas que hacían referencia a consecuencias negativas del consumo y se le daba el valor de 1 a cada respuesta afirmativa, se sumaban las respuestas afirmativas por factor y se dividía entre la cantidad de participantes que consumían alcohol.

Se utilizó el ANOVA de una vía para comparar el AUC obtenido de los diferentes grupos de consumo de alcohol. Asimismo, un ANOVA de medidas repetidas para evaluar el AUC obtenido en las tareas experimentales dentro de cada grupo de consumo de alcohol. La correlación de Pearson se llevó a cabo para evaluar la asociación entre todas las tareas de descuento. Con la correlación de Spearman se evaluó la asociación de las tareas de descuento con el patrón de consumo de alcohol y las consecuencias negativas del consumo. Los análisis se realizaron considerando un nivel de confianza del 0.05 .

\section{Resultados}

\section{Características de los participantes}

En la Tabla 1 se presentan los datos sociodemográficos de los participantes. Se muestra el porcentaje y media de las diversas variables en función del consumo de alcohol reportado. La Tabla 1 muestra que la cantidad de participantes, el porcentaje de hombres y porcentaje de quienes estudian y trabajan fue menor en el grupo de nulo consumo de alcohol. Asimismo, el grupo de nulo consumo mantiene un mayor porcentaje de mujeres.

Tabla 1. Datos sociodemográficos y agrupación de los participantes

\begin{tabular}{|c|c|c|c|}
\hline & $\begin{array}{l}\text { Nulo consumo de al- } \\
\text { cohol }(n=10)\end{array}$ & $\begin{array}{l}\text { Bajo consumo de al- } \\
\text { cohol }(n=33)\end{array}$ & $\begin{array}{l}\text { Consumo excesivo de al- } \\
\text { cohol }(n=23)\end{array}$ \\
\hline Hombres (\%) & 30 & 52 & 52 \\
\hline Solteros (\%) & 90 & 100 & 100 \\
\hline Casa propia (\%) & 90 & 87 & 85 \\
\hline Rentan casa (\%) & 10 & 13 & 15 \\
\hline Trabajan y estudian (\%) & 10 & 26 & 45 \\
\hline Solo estudian (\%) & 90 & 74 & 55 \\
\hline Edad (años) & 19.4 & 20.3 & 20.7 \\
\hline Ingresos familiares & $\$ 8,389$ & $\$ 8,984$ & $\$ 9,667$ \\
\hline
\end{tabular}

Nota.

Un participante del grupo de nulo consumo no especificó su estado civil. Los ingresos familiares reportados eran mensuales y en pesos mexicanos.

\section{Datos sistemáticos y asistemáticos}

Considerando todas las condiciones experimentales de la tarea monetaria, se observó un total de 219 datos sistemáticos (83\%) y 45 datos no sistemáticos (17\%). Del total de los datos asistemáticos, el $64 \%$ correspondió al criterio 1. En relación con la tarea con recompensas que implicaban salud, se observó un total de 206 datos sistemáticos (78\%) y 58 datos no sistemáticos (22\%). Del total de los datos asistemáticos, el 66\% correspondió al criterio 1 . En ambas tareas el porcentaje de datos asistemáticos es consistente con lo reportado en estudios previos (Smith et al., 2018).

\section{Efecto cero y de magnitud en función del consumo}

La Figura 1 muestra AUC de las condiciones con recompensas monetarias en función del consumo de alcohol. En el eje $y$ se puede identificar el valor descontado de la recompensa (normalizado) y en el eje $x$ se puede observar las 
condiciones experimentales en cada grupo de consumo de alcohol. Un primer resultado observable en la gráfica es una tendencia de los participantes a descontar más (menor AUC) conforme la cantidad de consumo de alcohol aumenta. Así, puede observarse una tendencia a registrar mayor AUC en el grupo de nulo consumo, seguido del grupo de bajo consumo de alcohol y, por último, el grupo de consumo excesivo de alcohol, esta tendencia se observa en todas las condiciones experimentales. No obstante, solo se encontraron diferencias significativas entre el grupo de nulo consumo y el grupo de consumo excesivo en las condiciones cero implícito $\$ 2,000$ y cero explícito $\$ 2,000$. Un ANOVA de una vía mostró diferencias significativas en el resultado señalado: cero implícito $\$ 2,000 \mathrm{~F}(2,63)=3.497, \mathrm{p}$ $<0.05$; cero explicito $\$ 2,000 F(2,63)=6.094, p<0.01$.

Un segundo resultado que muestra la Figura 1 es el efecto de hacer explícito el cero. Los hallazgos sugieren que tanto con la magnitud de $\$ 2,000$ como con la de $\$ 15,000$, el grado de descuento fue menor al hacer explícito el cero en los tres grupos de consumo. Finalmente, el efecto de magnitud se observó con los dos tipos de tarea (cero implícito y cero explícito) independientemente del nivel de consumo de alcohol. Un ANOVA de medidas repetidas de 2 (tipos de tarea) $\times 2$ (dominios) $\times 2$ (magnitudes) como factores intra-sujeto mostró un efecto significativo, independiente del nivel de consumo, del tipo de tarea, $F(1,63)=43.809, p<0.001, n_{p}^{2}=0.410$; del tipo de dominio, $F(1,63)=$ $15.140, p<0.001, n_{p}^{2}=0.194$; de la magnitud, $F(1,63)=33.082, p<0.001, n_{p}^{2}=0.344$. No obstante, se observó una interacción significativa entre el tipo de tarea y dominio, $F(1,63)=4$. 902, $p=0.030, n_{p}{ }_{p}=0.072$; entre la magnitud y el dominio, $F(1,63)=8.243, p=0.006, n_{p}{ }_{p}=0.116$, pues los efectos del tipo de tarea y magnitud de la recompensa solo se observaron al emplear dinero, pero no con recompensas de salud (ver Figura 2).

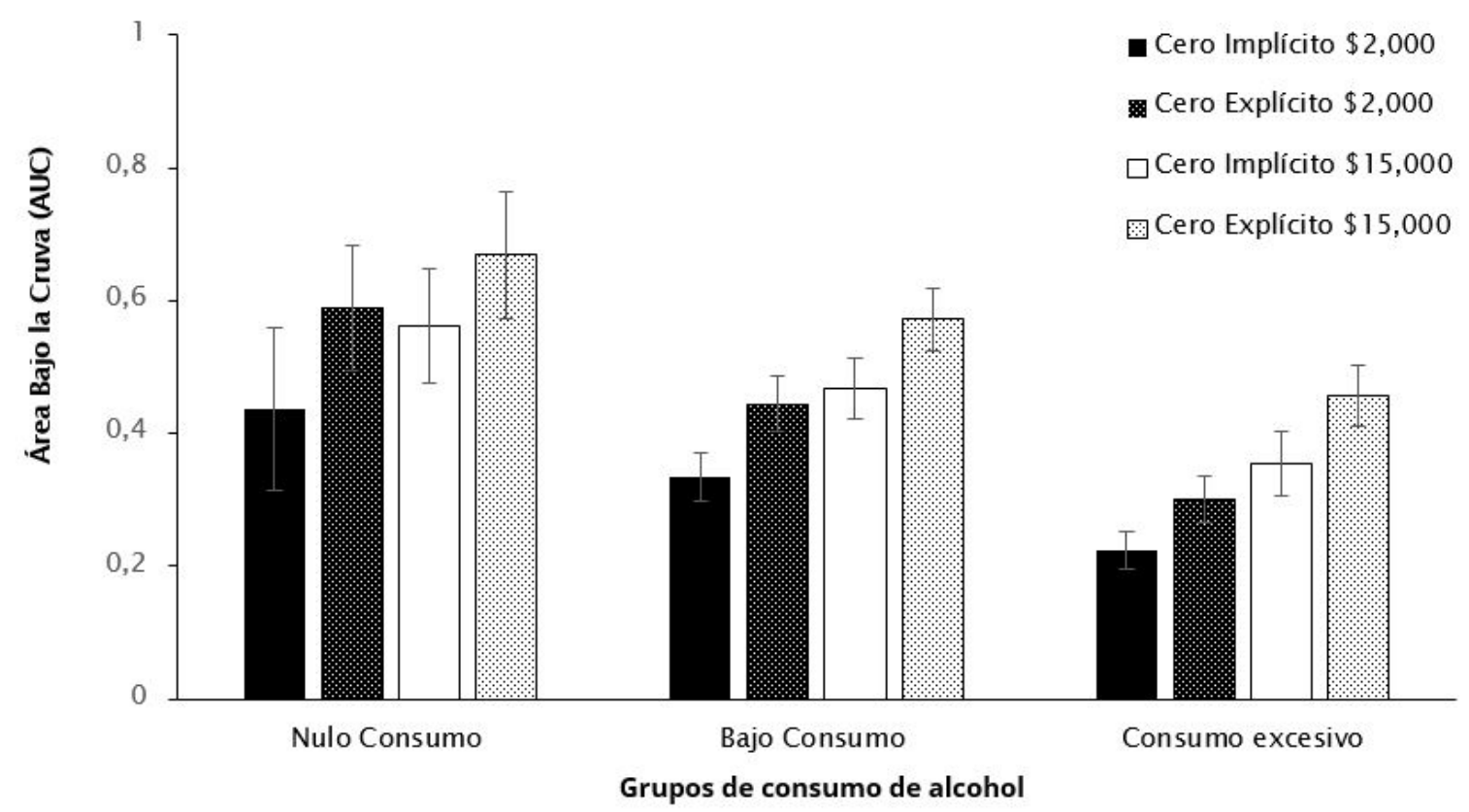

Figura 1. Efecto cero implícito y explícito con recompensas monetarias.

Nota. Área bajo la curva de las condiciones experimentales con recompensas monetarias en función del nivel de consumo de alcohol.

La Figura 2 muestra el AUC obtenido con las recompensas de salud en función del consumo de alcohol. Los datos muestran poca diferencia entre todas las condiciones de salud independientemente del nivel de consumo de alcohol. Asimismo, el AUC es ligeramente mayor cuando la recompensa es de 48 meses de salud versus 12 meses de salud y cuando la condición hacia explícito el cero en los tres grupos de consumo de alcohol, pero ninguna de las diferencias fue estadísticamente significativa. 


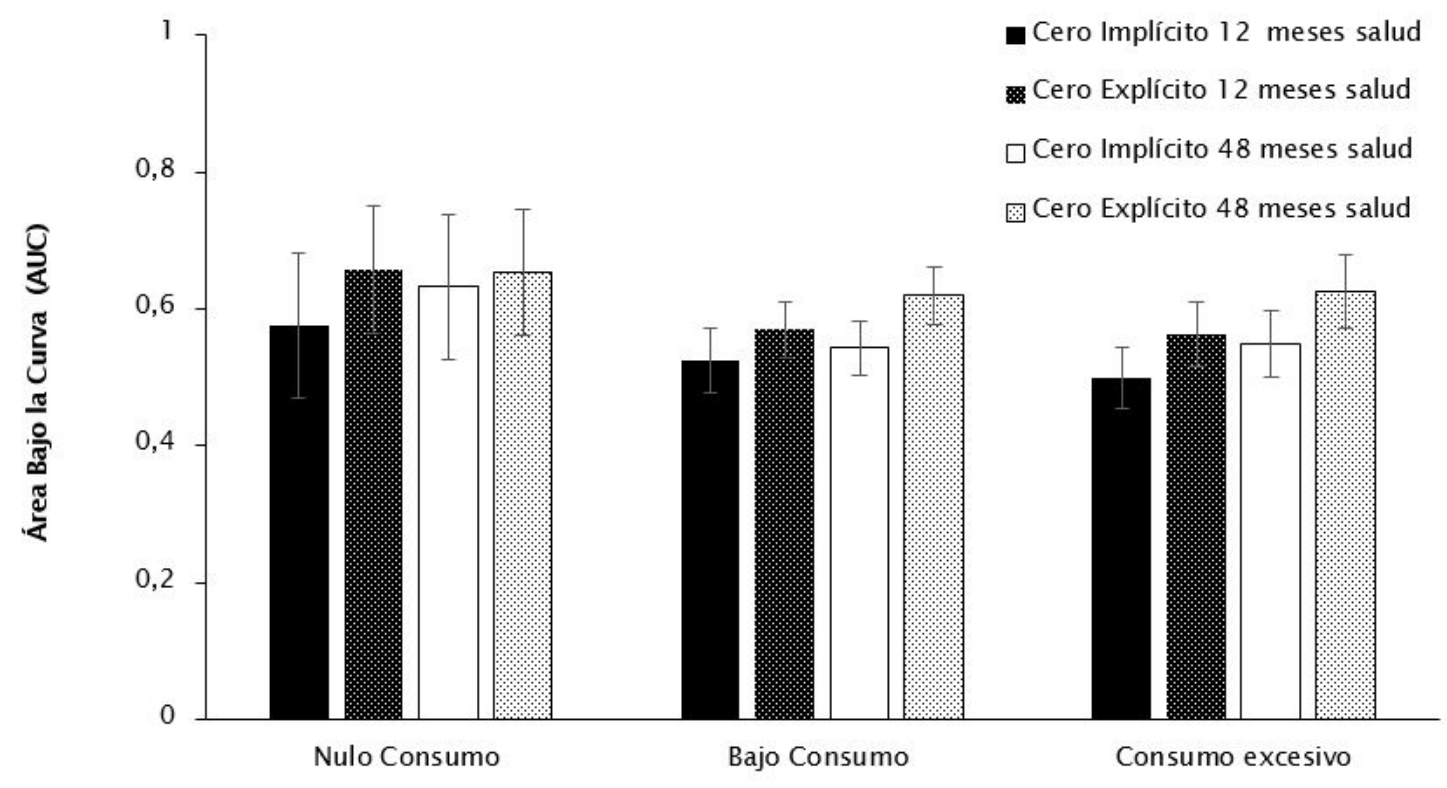

Grupos de consumo de alcohol

Figura 2. Efecto cero implícito y explícito en recompensas de salud

Nota. Área bajo la curva de las condiciones experimentales con recompensas de salud en función del nivel de consumo de alcohol.

\section{Consecuencias negativas: relación con el descuento y patrón de consumo}

La Tabla 2 muestra el promedio de consecuencias negativas obtenidas de los participantes que reportan consumir alcohol en al algún momento de la vida. Como puede constatarse, se reportaron pocas consecuencias, situación más evidente en el caso de aquellas consecuencias experimentadas en los últimos seis meses, razón por la que no se presentan.

Tabla 2. Media y desviación estándar de consecuencias negativas del consumo de alcohol.

\begin{tabular}{ll}
\hline Factores (en la vida) & (DE) \\
\hline Físicas, sociales y económicas & $2.6(\mathrm{DE}=1.97)$ \\
Asociadas al rol e interpersonales & $0.5(\mathrm{DE}=0.179)$ \\
Asociadas a la adicción & $0.375(\mathrm{DE}=0.58)$ \\
Transgresión de normas legales y convencionales & $0.196(\mathrm{DE}=0.77)$ \\
\hline
\end{tabular}

La Tabla 3 muestra la correlación Spearman entre las tareas de descuento temporal, el patrón de consumo de alcohol (cantidad y frecuencia de consumo de alcohol al mes) y las consecuencias negativas del consumo en la vida. En cuanto al patrón de consumo de alcohol, hubo una correlación negativa débil con tres de las cuatro condiciones experimentales de dinero, obteniendo solo la condición de cero explícito de $\$ 15,000$ un valor de $p<0.01$. En la cantidad de consumo de alcohol al mes solo hubo una correlación negativa débil con la condición cero explícito \$2,000 con un valor de $p<0.05$. Respecto a las consecuencias negativas, solo las que han pasado a lo largo de la vida se correlacionaron negativamente débil con la condición cero escondido de 48 meses de salud con un valor de $p<0.05$. 
Tabla 3. Correlación entre condiciones experimentales, consumo de alcohol y consecuencias negativas.

\begin{tabular}{|c|c|c|c|c|c|}
\hline \multirow[t]{2}{*}{ Mayor } & & \multicolumn{2}{|c|}{ Cero Implícito } & \multicolumn{2}{|c|}{ Cero Explicito } \\
\hline & & Menor & Mayor & Menor & \\
\hline \multirow[t]{5}{*}{ Dinero } & Patrón de consumo de Alcohol & $-.278 *$ & -.242 & $-.495 * *$ & $-.248^{*}$ \\
\hline & Cantidad de consumo al mes & -.227 & -.219 & -.170 & $-.296 *$ \\
\hline & Frecuencia de consumo al mes & -.230 & -.255 & -.186 & -.133 \\
\hline & Consecuencias negativas en la vida & -.153 & -.218 & -.130 & -.138 \\
\hline & Consecuencias negativas los últimos 6 meses & -.180 & -.152 & -.103 & -.121 \\
\hline \multirow[t]{5}{*}{ Salud } & Patrón de consumo de alcohol & -.093 & -.089 & -.095 & -.027 \\
\hline & Cantidad de consumo al mes & -.025 & -.033 & -.054 & -.041 \\
\hline & Frecuencia de consumo al mes & -.215 & -.161 & -.031 & -.078 \\
\hline & Consecuencias negativas en la vida & $-.300 *$ & -.239 & -.139 & -.149 \\
\hline & Consecuencias negativas los últimos 6 meses & .077 & -.144 & -.058 & -.030 \\
\hline
\end{tabular}

Notas. El nivel de alcohol se refiere a: (1) nulo consumo de alcohol; (2) bajo consumo de alcohol (menos de 5 copas para hombres y 4 para mujeres; solo un consumo al mes); (3) consumo excesivo (igual o mayor a 4 o 5 copas en más de una ocasión al mes para mujeres y hombres, respectivamente).

*Valor de $p<0.05$

$* *$ Valor de $p<0.01$

\section{Correlación entre dominios}

La Tabla 4 muestra la correlación de Pearson entre las condiciones experimentales. Los datos sugieren una correlación positiva estadísticamente significativa entre todas las condiciones de descuento, la correlación fue más fuerte para las condiciones del mismo dominio y la asociación tendió a ser más débil cuando se analizaban las condiciones experimentales entre dominios.

Tabla 4. Correlación entre tareas en ambos dominios.

\begin{tabular}{|c|c|c|c|c|c|c|c|}
\hline & $\begin{array}{l}\text { Cero } \\
\text { implícito } \\
\$ 2,000\end{array}$ & $\begin{array}{l}\text { Cero } \\
\text { implícito } \\
\$ 15,000\end{array}$ & $\begin{array}{l}\text { Cero } \\
\text { explícito } \\
\$ 2,000\end{array}$ & $\begin{array}{l}\text { Cero } \\
\text { explícito } \\
\$ 15,000\end{array}$ & $\begin{array}{l}\text { Cero } \\
\text { implícito } \\
12 \text { meses }\end{array}$ & $\begin{array}{l}\text { Cero } \\
\text { implícito } \\
48 \text { meses }\end{array}$ & $\begin{array}{l}\text { Cero explícito } \\
12 \text { meses }\end{array}$ \\
\hline $\begin{array}{l}\text { Cero implícito } \\
\$ 2,000\end{array}$ & - & & & & & & \\
\hline $\begin{array}{l}\text { Cero implícito } \\
\$ 15,000\end{array}$ & $.696 * *$ & - & & & & & \\
\hline $\begin{array}{l}\text { Cero explícito } \\
\$ 2,000\end{array}$ & $.775^{* *}$ & $.695^{* *}$ & - & & & & \\
\hline $\begin{array}{l}\text { Cero explícito } \\
\$ 15,000\end{array}$ & $.721^{* *}$ & $.794 * *$ & $.798^{* *}$ & - & & & \\
\hline
\end{tabular}




\begin{tabular}{|c|c|c|c|c|c|c|c|}
\hline $\begin{array}{l}\text { Cero implícito } \\
12 \text { meses }\end{array}$ & $.360 *$ & $.371^{*}$ & $.310^{*}$ & $.388^{* *}$ & - & & \\
\hline $\begin{array}{l}\text { Cero implícito } \\
48 \text { meses }\end{array}$ & $.262^{*}$ & $.263 *$ & $.264^{*}$ & $.323 *$ & $.754^{* *}$ & - & \\
\hline $\begin{array}{l}\text { Cero explícito } \\
12 \text { meses }\end{array}$ & $.305^{*}$ & $.365^{*}$ & $.296^{*}$ & $.336^{* *}$ & $.816^{* *}$ & $.796 * *$ & - \\
\hline $\begin{array}{l}\text { Cero explícito } \\
48 \text { meses }\end{array}$ & $.273^{*}$ & $.288^{*}$ & $.310 *$ & $.418^{* *}$ & $.665^{* *}$ & $.796 * *$ & $.714^{* *}$ \\
\hline
\end{tabular}

*Valor de $p<0.05$

$* *$ Valor de $p<0.01$

\section{Discusión}

El objetivo principal de la investigación fue evaluar si el grado de descuento temporal de jóvenes con diferentes patrones de consumo de alcohol disminuía cuando la situación experimental explicitaba el costo de oportunidad de las alternativas en dominios de dinero y salud. Los resultados principales mostraron (1) una tendencia a descontar la recompensa demorada en mayor grado conforme más se consume alcohol, pero únicamente en las condiciones experimentales que implican recompensas monetarias; (2) al hacer explícito el cero se descontaron menos las recompensas monetarias independientemente del nivel de consumo, pero no fue el caso de las recompensas de salud; (3) igualmente, en la tarea monetaria tanto con el cero implícito como con el cero explícito el descuento de una recompensa mostró ser dependiente de la magnitud; (4) solo una condición experimental se correlacionó negativamente con las consecuencias negativas del consumo.

Consistente con estudios previos, la tasa de descuento parece ser un predictor del consumo de alcohol. Por ello, identificar variables que reduzcan la tasa de descuento y ayuden a promover el consumo controlado o la abstinencia constituye una tarea fundamental. En este sentido, el efecto cero puede ser una manipulación que ayude a mejorar las decisiones de las personas. Empleando recompensas monetarias, jóvenes con distintos niveles de consumo de alcohol prefieren en mayor medida recompensas demoradas al hacer explícito el costo de elegir la recompensa inmediata, por lo que promover el análisis de los costos puede ser una estrategia útil para distintos grupos de personas.

No obstante, las diferencias entre los grupos que consumen alcohol y quienes no lo hacen parece mantenerse aun explicitando el costo de oportunidad. Esto puede indicar que las diferencias en el nivel de impulsividad asociadas a distintos grados de consumo de alcohol no necesariamente obedecen exclusivamente a que las personas no consideren el costo de sus elecciones. Sin embargo, en la presente manipulación solo se señalaba la no recepción de una recompensa, futuros trabajos pueden explorar si es posible que una persona que bebe alcohol en exceso estaría menos dispuesta a continuar con su patrón de consumo si consideran costos adicionales de su preferencia por el alcohol (e.g., no ahorrar, experimentar consecuencias negativas en el corto y largo plazo, etc.).

La generalidad del efecto cero es limitada al no observarse con recompensas de salud en ninguno de los tres grupos bajo el procedimiento empleado. Primeramente, los resultados sugieren un efecto de techo en las cuatro condiciones de salud. Posiblemente ambas magnitudes de salud se valoren como recompensas de gran magnitud y eso limite la influencia del efecto cero, por lo que no se puede asegurar la ausencia de los efectos en el dominio de salud. Futuros trabajos deberían de realizar un análisis paramétrico donde se utilicen diferentes magnitudes de salud.

Adicionalmente, los resultados en el dominio de salud se pueden interpretar en términos de lo que los participantes consideran que "debían elegir". El estudio de Nomicos et al. (2020) muestra que la tasa de descuento cambia en función de si las situaciones se enmarcan como una preferencia o una obligación. Los resultados del estudio mencionado muestran un menor grado de descuento de las recompensas cuando la situación preguntaba “¿Cuál debería 
elegir?” en comparación con la pregunta “¿Cuál me gustaría elegir?”. Aunque en nuestro estudio no se realizó la pregunta sobre lo que "debería elegir", el dominio de salud puede favorecer que los mayores beneficios de la salud sean percibidos por los participantes como algo que "debe elegirse", pues la salud suele ser descrita como algo valioso.

Una tercera posibilidad es que los participantes del presente estudio prefirieron esperar más por mayores beneficios de salud por que tienen poca experiencia con los costos de cuidar la salud. Es posible que el descuento de recompensas de salud cambie si los participantes tienen o no una enfermedad. En el presente estudio se descartó a jóvenes que manifestaron tener una enfermedad crónica para controlar dicha variable y posibilitar una mejor comprensión de las variables estudiadas. No obstante, una persona con algún padecimiento podría reconocer los costos del cuidado de la salud que cotidianamente influyen en la preferencia por beneficios inmediatos, mismos que en el presente estudio no son considerados en la situación descrita. Es indispensable realizar estudios donde se compare el descuento de recompensas de salud en población sana y con alguna enfermedad para conocer si padecer alguna condición permite ponderar con mayor precisión los costos y beneficios de seguir un tratamiento.

Pocos estudios han buscado explorar la relación entre la tasa de descuento y las consecuencias negativas asociadas al consumo. En el presente estudio se encontró asociación negativa entre el cero implícito de 48 meses de salud y las consecuencias negativas del consumo de alcohol que pasan a lo largo de la vida. El resultado concuerda con el estudio de Dennhardt y Murphy (2011) donde indican que la población afroamericana (un subgrupo del estudio) con consumo problemático de alcohol reportó mayor cantidad de consecuencias negativas asociadas a un mayor grado de descuento. Sin embargo, en el presente estudio, los participantes reportaron pocas consecuencias negativas, esto podría explicar porque solo se asoció a una condición experimental. La relación estudiada podría ser más evidente con población con historial de dependencia al alcohol o que consumen sustancias ilegales. Sería útil en contexto clínicos identificar si la conducta impulsiva participa no solo en el consumo de sustancias, sino en el desarrollo de consecuencias negativas (e.g., físicas, económicas y legales).

Finalmente, el efecto de magnitud se replicó en tareas monetarias tanto con el cero implícito como explícito. Múltiples estudios muestran que recompensas monetarias más grandes se descuentan menos en comparación con recompensas más pequeñas (Ballard et al., 2017; Berry et al., 2017; Herman et al., 2020; Myerson et al., 2017; Sawicki et al., 2019). Asimismo, nuestro estudio concuerda con los hallazgos de Petry (2001) respecto a que las personas que consumen alcohol son más sensibles a la recompensa demorada cuando esta es de gran magnitud. Los resultados muestran generalidad del efecto de magnitud a situaciones en las que el cero es explícito, tanto en los jóvenes que no consumen alcohol como con aquellos que reportaron consumo controlado y excesivo.

\section{Reconocimiento}

El primer autor agradece al Consejo Nacional de Ciencia y Tecnología (CONACyT) por la beca otorgada para el desarrollo del presente proyecto. Asimismo, el segundo autor agradece al CONACYT el apoyo para el desarrollo del proyecto 281949 del cual el presente trabajo forma parte.

\section{Conflicto de intereses}

Los autores declaran no tener conflicto de intereses.

\section{Referencias}

Adams, S., Attwood, A. S., \& Munafò, M. R. (2017). Drinking status but not acute alcohol consumption influences delay discounting. Human Psychopharmacology: Clinical and Experimental, 32, 1-7. https://doi.org/10.1098/ $\underline{\text { rsos. } 170045}$

Aisline, G. (1975). Specious reward: A behavioral theory of impulsiveness and impulse control. Psychological Bulletin, 82, 463-406. https://doi.org/10.1037/h0076860 
Valenzuela-Reyes, J.L., Reyes-Huerta, H.E., dos Santos, C.V. y González-Torres, M.L.

Amlung, M., Vedelago, L., Acker, J., Balodis, I., \& MacKillop, J. (2017). Steep delay discounting and addictive behavior: A meta-analysis of continuous associations. Addiction, 112, 51-62. https://doi.org/10.1037/h0076860

Athamneh, L. N., Brown, J., Stein, J. S., Gatchalian, K. M., LaConte, S. M., \& Bickel, W. K. (2021). Future thinking to decrease real-world drinking in alcohol use disorder: Repairing reinforcer pathology in a randomized proof-ofconcept trial. Experimental and Clinical Psychopharmacology. Advance online publication. https://doi.org/10.1037/ pha0000460

Athamneh, L. N., DeHart, W. B., Pope, D., Mellis, A. M., Snider, S. E., Kaplan, B. A., \& Bickel, W. K. (2019). The phenotype of recovery III: Delay discounting predicts abstinence self-efficacy among individuals in recovery from substance use disorders. Psychology of Addictive Behaviors, 33, 310-318. http://dx.doi.org/10.1037/adb0000460

Bailey, A., Gerst, K., \& Fin, P. (2018). Delay discounting of losses and rewards in alcohol Use Disorder: The Effect of Working Memory Load. Psychology of Addictive Behaviors, 32, 197-204. https://doi.org/10.1111/acer.13472

Ballard, I. C., Kim, B., Liatsis, A., Aydogan, G., Cohen, J. D., \& McClure, S. M. (2017). More is meaningful: The magnitude effect in intertemporal choice depends on self-control. Psychological Science, 28, 1443-1454. https://doi. org/10.1177/0956797617711455

Berry, M. S., Friedel, J. E., DeHart, W. B., Mahamane, S., Jordan, K. E., \& Odum, A. L. (2017). The value of clean air: Comparing discounting of delayed air quality and money across magnitudes. The Psychological Record, 67, 137-148. https://doi.org/10.1007/s40732-017-0233-4

Bickel, W. K., Odum, A. L., \& Madden, G. J. (1999). Impulsivity, and cigarette smoking: Delay discounting un current, never, and ex-smokers. Psichopharmacology, 146, 337-454. https://doi.org/10.1007/PL00005490

Chapman, G. B. (1996). Temporal discounting and utility for health and money. Journal of Experimental Psychology: Learning, Memory, and Cognition, 22, 771-791. https://doi.org/10.1037/0278-7393.22.3.771

De Wit, H., \& Richards, J. B. (2004). Dual Determinants of Drug Use in Humans: Reward and Impulsivity. In R. A. Bevins \& M. T. Bardo (Eds.), Motivational factors in the etiology of drug abuse. Vol. 50 of the Nebraska Symposium on Motivation (pp. 19-55). University of Nebraska Press.

Dennhardt, A. A., \& Murphy, J. G. (2011). Associations between depression, distress tolerance, delay discounting, and alcohol-related problems in European American and African American college students. Psychology of Addictive Behaviors, 25, 595-604. http://dx.doi.org/10.1037/a0025807

Field, M., Christiansen, P., Cole, J., \& Goudie, A. (2007). Delay discounting and the alcohol Stroop in heavy drinking adolescents. Addiction, 102, 579-586. https://doi.org/10.1111/i.1360-0443.2007.01743.

Gerst, K. R., Gunn, R. L., \& Finn, P. R. (2017). Delay discounting of losses in alcohol use disorders and antisocial psychopathology: Effects of a working memory load. Alcoholism: Clinical and Experimental Research, 41, 1768-1774. https://doi.org/10.1111/acer.1347

Gowin, J., Sloan, M. E., Swan, J. E., Momenan, R., \& Ramchandani, V. A. (2019). The relationship between delay discounting and alcohol dependence in individuals with and without comorbid psychopathology. Psychopharmacology, 236, 775-785. https://doi.org/10.1007/s00213-018-5113-3

Harman, M., Kodak, T., \& McKerchar, T. (2020). Effects of reward magnitude frames on measures of delay discounting in a hypothetical money scenario. Journal of the Experimental Analysis of Behavior, 114, 193-202. https://doi. org/10.1002/jeab.620 
Du, W., Green, L., \& Myerson, J. (2002). Cross-cultural comparisons of discounting delayed and probabilistic rewards. The Psychological Record, 52, 479-492. https://doi.org/10.1007/BF03395199.

Johnson, M. W., \& Bickel, W. K. (2008). An algorithm for identifying nonsystematic delay-discounting data. Experimental and Clinical Psychopharmacology, 16, 264-274. https://doi.org/10.1037/1064-1297.16.3.264

Koffarnus, M. N., \& Bickel, W. K. (2014). A 5-trial adjusting delay discounting task: Accurate discount rates in less than one minute. Experimental and Clinical Psychopharmacology, 22, 222-228. https://doi.org/10.1037/a0035973

Kirby, K. N., Petry, N. M., \& Bickel, W. K. (1999). Heroin addicts have higher discount rates for delayed rewards than non-drug-using controls. Journal of Experimental Psychology: General, 128, 78-87. https://doi.org/10.1037/0096$\underline{3445.128 .1 .78}$

Magen, E., Dweck, C., \& James, G. (2008). The hidden-zero effect. Representing a single choice as an extended sequence reduces impulsive choice. Association for Psychological Science, 19, 648-649. https://doi.org/10.1111/ j.1467-9280.2008.02137.x

Mayhew, M. J., Byrne, J. M., Powell, J. H., \& Meynen, T. (2020). Are hazardous drinkers more impulsive than light drinkers? A comprehensive assessment in young adults. Alcohol, 84, 9-20. https://doi.org/10.1016/j.alco$\underline{\text { hol.2019.09.00 }}$

Mitchell, S. H. (2019). Linking delay discounting and substance use disorders: Genotypes and phenotypes. Perspectives on Behavioral Science, 42, 419-432. https://doi.org/10.1007/s40614-019-00218-x.

Morrison, K. L., Madden, G. J., Odum, A. L., Friedel, J. E., \& Twohig, M. P. (2014). Altering impulsive decision making with an acceptance-based procedure. Behavior Therapy, 45, 630-639. https://doi.org/10.1016/i.beth.2014.01.001

Myerson, J., Baumann, A. A., \& Green, L. (2017). Individual differences in delay discounting: Differences are quantitative with gains, but qualitative with losses. Journal of Behavioral Decision Making, 30, 359-372. https://doi. org/10.1002/bdm.1947

Myerson, J., Green, L., Van Den Berk-Clark, C., \& Grucza, R. A. (2015). Male, but not female, alcohol-dependent African Americans discount delayed gains more steeply than propensity-score matched controls. Psychopharmacology, 232, 4493-4503. https://doi.org/10.1007/s00213-015-4076-x

Naudé, G. P., Kaplan, B. A., Reed, D. D., Henley, A. J., \& DiGennaro Reed, F. D. (2018). Temporal framing and the hidden-zero effect: rate-dependent outcomes on delay discounting. Journal of the Experimental Analysis of Behavior, 109, 506-519. https://doi.org/10.1002/jeab.328

Nomicos, L., Jacobs, K.W. \& Locey, M.L. (2020). The effects of obligatory and preferential frames on delay discounting. Analysis Verbal Behavior, 36, 74-86. https://doi.org/10.1007/s40616-020-00127-0

Odum, A. L., Becker, R. J., Haynes, J. M., Galizio, A., Frye, C. C., Downey, H., \& Perez, D. M. (2020). Delay discounting of different outcomes: Review and theory. Journal of the Experimental Analysis of Behavior, 113, 657-679. https:// doi.org/10.1002/jeab.589

Rachlin, H. (1995). Self-control: Beyond commitment. Behavioral and Brain Sciences, 18, 109-121. https://doi. org/10.1017/S0140525X00037602

Radu, P. T., Yi, R., Bickel, W. K., Gross, J. J., \& McClure, S. M. (2011). A mechanism for reducing delay discounting by 
altering temporal attention. Journal of the Experimental Analysis of Behavior, 96, 363-385. https://doi.org/10.1901/ jeab.2011.96-363

Sawicki, P., Markiewicz, Ł., \& Białek, M. (2020). Magnitude effect contributes to the domain specificity in delay discounting. Journal of Behavioral Decision Making, 33, 323-332. https://doi.org/10.1002/bdm.2159

Salazar, M. L., Vacio, M. A., López, M. A., \& Sánchez, F. M. (2012). Adaptación del Young Adult Alcohol Problem Screening Test (YAAPST) con estudiantes universitarios de México. Investigación y Ciencia, 20, 44-52.

Scholten, H., Scheres, A., de Water, E., Graf, U., Granic, I., \& Luijten, M. (2019). Behavioral trainings and manipulations to reduce delay discounting: A systematic review. Psychonomic Bulletin \& Review, 26, 1803-1849. https://doi. org/10.3758/s13423-019-01629-2

Secretaria de Salud, Consejo Nacional contra las Adicciones, Instituto Nacional de Psiquiatría, Instituto Nacional de Salud Pública (2016). Encuesta Nacional de Adicciones. Cuestionario Individual. Instrumento no publicado. https:// encuestas.insp.mx/ena/encodat2017/cuestionario encodat individual 2016 2017.pdf

Snider, S. E., DeHart, W. B., Epstein, L. H., \& Bickel, W. K. (2019). Does delay discounting predict maladaptive health and financial behaviors in smokers? Health Psychology, 8, 21-28. https://doi.org/10.1037/hea0000695

Smith, K. R, Lawyer, S. R., \& Swift, J. K. (2018). A Meta-Analysis of nonsystematic responding in delay and probability reward discounting. Experimental and Clinical Psychopharmacology, 26, 94-107. https://doi.org/10.1037/ pha0000167

Stam, C. H., van der Veen, F. M., \& Franken, I. H. (2020). Individual differences in time estimation are associated with delay discounting and alcohol use. Current Psychology, 1-10. https://doi.org/10.1007/s12144-020-00899-7

Stanger, C., Ryan, S. R., Fu, H., Landes, R. D., Jones, B. A., Bickel, W. K., \& Budney, A. J. (2012). Delay discounting predicts adolescent substance abuse treatment outcome. Experimental and Clinical Psychopharmacology, 20, 205-212. https://doi.org/10.1037/a0026543

Takahashi, T., Ohmura, Y., \& Radford, M. (2009). Alcohol use and discounting of delayed and probabilistic gain and loss. Neuroendorinology Letters, 30, 749-752.

World Health Organization (2018). Global Status Report on Alcohol and Health. Geneva: World Health Organization. https://www.who.int/substance abuse/publications/global alcohol report/gsr 2018/en/

Wu, C. Y., \& He, G. B. (2012). The effects of time perspective and salience of possible monetary losses on intertemporal choice. Social Behavior and Personality: An International Journal, 40, 1645-1653. https://doi.org/10.2224/ $\underline{\text { sbp.2012.40.10.1645 }}$

\section{Apéndice}

\section{Descripción presentada en la tarea de descuento en el dominio de salud}

Descripción presentada a los participantes en la tarea de salud. Esta situación general se adaptó para evaluar el efecto cero agregando la no recepción de la alternativa no elegida, el resto fue exactamente igual. 
Imagine que la siguiente situación hipotética la ha tenido durante dos años y seguirá así durante el resto de su vida:

Debe tener mucho cuidado con los alimentos que come, por lo que pasa mucho tiempo haciendo un seguimiento de sus alimentos. Le resulta necesario visitar el baño con bastante frecuencia para orinar. A menudo se siente cansado y mareado. A veces tiene problemas para conciliar el sueño y en ocasiones tiene pesadillas cuando duerme. Su boca suele sentirse seca, y los alimentos no parecen tener tanto sabor como solían tener. No tiene tanto deseo sexual como antes, y no considera que el sexo sea tan agradable como solía serlo. Constantemente se siente enojado o irritado, y es difícil concentrarse.

Ahora imagine que existen dos tratamientos que pueden regresarle la salud momentáneamente. El tratamiento $A$ (Tx A) le traerá muchos MESES DE SALUD después de cierto tiempo y el Tratamiento B (Tx B) le traería menos MESES DE SALUD inmediatamente.

Tanto el tratamiento A como el tratamiento B podrán ser elegidos solo una vez en un periodo de 5 años. 